\title{
Spectral transformations of measures supported on the unit circle and the Szegó transformation
}

\author{
Luis Garza • Javier Hernández • \\ Francisco Marcellán
}

\begin{abstract}
In this paper we analyze spectral transformations of measures supported on the unit circle with real moments. The connection with spectral transformations of measures supported on the interval $[-1,1]$ using the Szegó transformation is presented. Some numerical examples are studied.
\end{abstract}

Keywords Measures on the unit circle - Orthogonal polynomials • Carathéodory functions $\cdot$ Spectral transformations $\cdot$ LU factorization

Mathematics Subject Classifications (2000) $42 \mathrm{C} 05 \cdot 15 \mathrm{~A} 23$

\section{Introduction}

Let $\mu$ be a positive, non trivial Borel measure, supported on a subset $E$ of the real line. The sequence of polynomials $\left\{p_{n}\right\}_{n \geqslant 0}$, with

$$
p_{n}(x)=\gamma_{n} x^{n}+\delta_{n} x^{n-1}+\ldots, \quad \gamma_{n}>0
$$

L. Garza $\cdot$ F. Marcellán $(\bowtie)$

Universidad Carlos III de Madrid, Avenida de la Universidad 30,

28911 Leganés, Madrid, Spain

e-mail: pacomarc@ing.uc3m.es

L. Garza

e-mail: lgaona@uat.edu.mx

L. Garza

Universidad Autónoma de Tamaulipas, Ciudad Victoria, Mexico

J. Hernández

Universidad Centroccidental Lisandro Alvarado, Barquisimeto, Venezuela

e-mail: jbenitez@ucla.edu.ve 
is said to be an orthonormal polynomial sequence associated with $\mu$ if

$$
\int_{E} p_{n}(x) p_{m}(x) d \mu(x)=\delta_{m, n}, \quad m, n \geqslant 0 .
$$

The corresponding monic orthogonal polynomials, i.e. with leading coefficient equal to 1 , are defined by

$$
P_{n}(x)=\frac{p_{n}(x)}{\gamma_{n}} .
$$

The sequence $\left\{p_{n}\right\}_{n \geqslant 0}$ satisfies the following three-term recurrence relation

$$
x p_{n}(x)=a_{n+1} p_{n+1}(x)+b_{n} p_{n}(x)+a_{n} p_{n-1}(x), \quad n \geqslant 0,
$$

with $a_{n}=\frac{\gamma_{n-1}}{\gamma_{n}}>0, n \geqslant 1$, and $b_{n}=\frac{\delta_{n}}{\gamma_{n}}-\frac{\delta_{n+1}}{\gamma_{n+1}}, n \geqslant 0$. The matrix representation of (1) is

$$
x p(x)=J p(x),
$$

where $p(x)=\left[p_{0}(x), p_{1}(x), \ldots\right]^{t}$ and $J$ is a tridiagonal symmetric matrix

$$
J=\left(\begin{array}{ccccc}
b_{0} & a_{1} & 0 & 0 & \cdots \\
a_{1} & b_{1} & a_{2} & 0 & \cdots \\
0 & a_{2} & b_{2} & a_{3} & \ddots \\
0 & 0 & a_{3} & b_{3} & \ddots \\
\vdots & \vdots & \ddots & \ddots & \ddots
\end{array}\right)
$$

which is called in the literature Jacobi matrix [2]. There exists a similar expression to (1) using the monic orthogonal polynomials. In such a case, the matrix representation is

$$
x P(x)=\tilde{J} P(x),
$$

where $P(x)=\left[P_{0}(x), P_{1}(x), \ldots\right]^{t}$ and $\tilde{J}$ is a tridiagonal matrix

$$
\tilde{J}=\left(\begin{array}{ccccc}
b_{0} & 1 & 0 & 0 & \cdots \\
a_{1}^{2} & b_{1} & 1 & 0 & \cdots \\
0 & a_{2}^{2} & b_{2} & 1 & \ddots \\
0 & 0 & a_{3}^{2} & b_{3} & \ddots \\
\vdots & \vdots & \ddots & \ddots & \ddots
\end{array}\right)
$$

which is called monic Jacobi matrix.

The functions

$$
S_{n}(x)=\int_{E} \frac{p_{n}(t) d \mu(t)}{x-t}
$$

constitute a second solution to the difference equation

$$
x y_{n}=a_{n+1} y_{n+1}+b_{n} y_{n}+a_{n} y_{n-1}
$$


and they are called second kind functions associated with $\mu$. The Stieltjes function

$$
S(x)=S_{0}(x)=\int_{E} \frac{d \mu(t)}{x-t}
$$

has a significant relevance in the theory of orthogonal polynomials and admits the following series expansion at infinity

$$
S(x)=\sum_{k=0}^{\infty} \frac{\mu_{k}}{x^{k+1}}
$$

where $\mu_{k}$ are the moments associated with $\mu$ given by

$$
\mu_{k}=\int_{E} x^{k} d \mu(x), \quad k \geqslant 0 .
$$

Given a measure $\mu$ supported on the real axis, some examples of perturbations of such a measure have been studied (see $[1,15]$ ). In particular, three canonical cases are considered:

1. The perturbation $d \tilde{\mu}=(x-\beta) d \mu, \beta \notin \operatorname{supp}(\mu)$, is the so-called canonical Christoffel transformation.

2. The perturbation $d \tilde{\mu}=d \mu+M_{r} \delta_{\beta}, \beta \notin \operatorname{supp}(\mu), M_{r} \in \mathbb{R}$, is the so-called canonical Uvarov transformation.

3. The perturbation $d \tilde{\mu}=\frac{d \mu}{x-\beta}+M_{r} \delta_{\beta}, \beta \notin \operatorname{supp}(\mu), M_{r} \in \mathbb{R}$, is the so-called canonical Geronimus transformation.

In [1], the monic Jacobi matrix associated with each of the three canonical perturbations in terms of the monic Jacobi matrix associated with $\mu$ is studied taking into account their $L U$ and $U L$ factorizations.

We will call a spectral rational transformation of a Stieltjes function $S(x)$, a transformation

$$
\tilde{S}(x)=\frac{A(x) S(x)+B(x)}{C(x) S(x)+D(x)},
$$

where $A(x), B(x), C(x)$, and $D(x)$ are polynomials in $x$. In the particular case when $C(x)=0$, the transformation is said to be linear. The three canonical perturbations mentioned above correspond to spectral linear transformations when they are expressed in terms of the Stieltjes functions.

In this paper we consider measures on the unit circle, defined from Borel measures supported on the interval $[-1,1]$ and we analyze the connection between spectral transformations of them. In particular, we focus our attention in the three canonical ones defined above. In Section 2, the background about orthogonal polynomials with respect to Borel measures supported on the unit circle is presented. In Section 3 we introduce the Szegó transformation from measures $\mu$ supported on the interval $[-1,1]$ to measures $\sigma$ supported on the unit circle such that their moments are real numbers. A connection between the $L U$ factorization of the Jacobi matrix associated with $\mu$ and the Verblunsky coefficients of the measure $\sigma$ is stated. Thus, a new algorithm to 
define the Verblunsky coefficients is deduced taking into account the entries of the matrices $L$ and $U$ of the factorization mentioned above. In Section 4 we describe the counterparts on the unit circle of the canonical spectral transformations on the real line, using the Szegó transformation. It is shown that the resulting transformations on the unit circle are of the same kind that those applied on the real line. Finally, in Section 5 some examples of spectral transformations on the unit circle are presented as well as the computation of the Verblunsky coefficients for the corresponding Borel measures using our algorithm. In fact, we show that these spectral transformations come from spectral transformations of the same kind on the real line, via the Szegó transformation.

\section{Orthogonal polynomials on the unit circle and spectral transformations}

Let $\sigma$ be a positive, non trivial Borel measure, supported on the unit circle $\mathbb{T}=\{z \in \mathbb{C}:|z|=1\}$. Then there exists a sequence $\left\{\varphi_{n}\right\}_{n \geqslant 0}$ of orthonormal polynomials

$$
\varphi_{n}(z)=\kappa_{n} z^{n}+\ldots, \quad \kappa_{n}>0,
$$

which satisfies

$$
\int_{-\pi}^{\pi} \varphi_{n}\left(e^{i \theta}\right) \overline{\varphi_{m}\left(e^{i \theta}\right)} d \sigma(\theta)=\delta_{m, n}, \quad m, n \geqslant 0 .
$$

The corresponding monic polynomials are defined by

$$
\Phi_{n}(z)=\frac{\varphi_{n}(z)}{\kappa_{n}} .
$$

The sequence $\left\{\Phi_{n}\right\}_{n \geqslant 0}$ also satisfies the following recurrence relations (see $[5,13,14])$

$$
\begin{aligned}
& \Phi_{n+1}(z)=z \Phi_{n}(z)+\Phi_{n+1}(0) \Phi_{n}^{*}(z), \\
& \Phi_{n+1}^{*}(z)=\Phi_{n}^{*}(z)+\overline{\Phi_{n+1}(0)} z \Phi_{n}(z),
\end{aligned}
$$

and the complex numbers $\left\{\Phi_{n}(0)\right\}_{n \geqslant 0}$ are called Verblunsky coefficients. Notice that $\left|\Phi_{n}(0)\right|<1$

$c_{k}$, the $k$-th moment associated with the measure $\sigma$, is defined by

$$
c_{k}=\int_{-\pi}^{\pi} e^{i k \theta} d \sigma(\theta)
$$

In these conditions, we can introduce an analytic function in terms of the moments $\left\{c_{n}\right\}_{n \geqslant 0}$ as follows

$$
F(z)=c_{0}+2 \sum_{k=1}^{\infty} c_{-k} z^{k} .
$$


$F(z)$ is analytic on the unit open disc and $\mathfrak{R e} F(z)>0$ in such a disk. $F(z)$ is called Carathéodory function and it can be represented as a Riesz-Herglotz transformation of $\sigma$ as follows

$$
F(z)=\int_{-\pi}^{\pi} \frac{e^{i \theta}+z}{e^{i \theta}-z} d \sigma(\theta) .
$$

We consider three cases of canonical transformations introduced in [3]

1. The perturbation $d \tilde{\sigma}=|z-\alpha|^{2} d \sigma,|z|=1, \alpha \in \mathbb{C}$, is the so-called canonical Christoffel transformation (see [10]).

2. The perturbation $d \tilde{\sigma}=d \sigma+M_{c} \delta_{\alpha}+\bar{M}_{c} \delta_{\bar{\alpha}^{-1}}, \alpha \in \mathbb{C}-\{0\}, M_{c} \in \mathbb{C}$, is the so-called Uvarov transformation (see [8]).

3. The perturbation $d \tilde{\sigma}=\frac{d \sigma}{|z-\alpha|^{2}},|z|=1$, and $|\alpha| \neq 1$ is the so-called Geronimus transformation (see $[4,6,9]$ ).

These three transformations correspond, in an analogue way to the real axis, to linear transformations of the corresponding Carathéodory functions. This approach was studied in [8].

\section{The Szegö transformation}

From a positive, non trivial Borel measure $\mu$, supported on $[-1,1]$, we can define a measure $\sigma$ in $[-\pi, \pi]$ as

$$
d \sigma(\theta)=\frac{1}{2}|d \mu(\cos \theta)|,
$$

so if $d \mu(x)=\omega(x) d x$, then

$$
d \sigma(\theta)=\frac{1}{2} \omega(\cos \theta)|\operatorname{sen} \theta| d \theta .
$$

If $\mu$ is a probability measure, i.e., $\int_{-1}^{1} d \mu=1$, then $\sigma$ is also a probability measure on the unit circle $\mathbb{T}=\{z \in \mathbb{C}:|z|=1\}$ and then there exists an orthonormal polynomial sequence that satisfies (2), as well as the corresponding monic orthogonal polynomials. In this case,

$$
\Phi_{n}(0) \in(-1,1), \quad n=1,2, \ldots
$$

There is a relation between the orthogonal polynomials associated with a measure $\mu$ supported on $[-1,1]$ and the orthogonal polynomial sequence associated with the measure $\sigma$ defined by (5), which is supported on the unit circle.

Theorem 1 [14] The monic orthogonal polynomial sequence $\left\{\Phi_{n}\right\}_{n \geqslant 0}$ on the unit circle associated with the measure $\sigma$ has real coefficients. In addition, if $x=\frac{\left(z+z^{-1}\right)}{2}$, then

$$
p_{n}(x)=\frac{\kappa_{2 n}}{2\left(\sqrt{1+\Phi_{2 n}(0)}\right)}\left[z^{-n} \Phi_{2 n}(z)+z^{n} \Phi_{2 n}(1 / z)\right] .
$$


On the other hand, the coefficients of the recurrence relations (1), (3), and (4) are related by [5]

$$
\begin{aligned}
& 2 a_{n}=\sqrt{\left[1-\Phi_{2 n}(0)\right]\left[1-\Phi_{2 n-1}^{2}(0)\right]\left[1+\Phi_{2 n-2}(0)\right]}, \quad n \geqslant 1 \\
& 2 b_{n}=\Phi_{2 n-1}(0)\left[1-\Phi_{2 n}(0)\right]-\Phi_{2 n+1}(0)\left[1+\Phi_{2 n}(0)\right], \quad n \geqslant 0 .
\end{aligned}
$$

There is another way to relate the coefficients of the recurrence relations, as follows

Theorem 2 [5] Let $\mu$ be a probability measure supported on the interval $[-1,1]$ and let $\sigma$ be the probability measure supported on the unit circle associated with $\mu$ via the Szegö transformation. Let $\left\{\Phi_{n}(0)\right\}_{n \geqslant 0}$ be the Verblunsky coefficients associated with $\sigma$ and $\left\{a_{n}^{2}\right\}_{n \geqslant 1},\left\{b_{n}\right\}_{n \geqslant 0}$ be the coefficients of the recurrence relation associated with the orthonormal polynomials with respect to $\mu$. Then, for $n \geqslant 0$

$$
\begin{aligned}
\Phi_{2 n}(0) & =w_{n}+v_{n}-1, \\
\Phi_{2 n+1}(0) & =\frac{w_{n}-v_{n}}{w_{n}+v_{n}},
\end{aligned}
$$

where

$$
\begin{gathered}
w_{n}=1-b_{n}-a_{n}^{2}\left(w_{n-1}\right)^{-1}, \\
v_{n}=1+b_{n}-a_{n}^{2}\left(v_{n-1}\right)^{-1},
\end{gathered}
$$

with the convention $w_{-1}^{-1}=v_{-1}^{-1}=0$.

These relations are called in the literature Inverse Geronimus Relations (see [5]). Notice that [13] shows a slightly different Inverse Geronimus Relation, because a normalization of the measure $\mu$ which is supported on the interval $[-2,2]$ is used.

On the other hand, if we define a sequence $\left\{u_{k}\right\}_{k \geqslant 1}$, with

$$
u_{k}=\frac{1}{2}\left(1-\Phi_{k}(0)\right)\left(1+\Phi_{k-1}(0)\right),
$$

we get

$$
\begin{aligned}
a_{k}^{2} & =u_{2 k} u_{2 k-1}, \quad k \geqslant 1 \\
b_{k}+1 & =u_{2 k}+u_{2 k+1}, \quad k \geqslant 0,
\end{aligned}
$$

and then we get a unique factorization

$$
\tilde{J}+I=L U
$$


where $I$ is the identity matrix, and $L$ and $U$ are lower and upper bidiagonal matrices, respectively, with

$$
L=\left(\begin{array}{ccccc}
1 & 0 & 0 & 0 & \cdots \\
u_{2} & 1 & 0 & 0 & \cdots \\
0 & u_{4} & 1 & 0 & \ddots \\
0 & 0 & u_{6} & 1 & \ddots \\
\vdots & \vdots & \ddots & \ddots & \ddots
\end{array}\right) \text { and } U=\left(\begin{array}{ccccc}
u_{1} & 1 & 0 & 0 & \cdots \\
0 & u_{3} & 1 & 0 & \cdots \\
0 & 0 & u_{5} & 1 & \ddots \\
0 & 0 & 0 & u_{7} & \ddots \\
\vdots & \vdots & \ddots & \ddots & \ddots
\end{array}\right)
$$

Thus, from (10)

$$
\Phi_{k}(0)=1-\frac{2 u_{k}}{1+\Phi_{k-1}(0)}
$$

or,

$$
\Phi_{k}(0)=1-\frac{2 u_{k}}{2-\frac{2 u_{k-1}}{2-\frac{2 u_{k-2}}{2-\frac{\ddots}{2-\frac{2 u_{2}}{2-2 u_{1}}}}}} .
$$

Therefore, we obtain a sequence that we can use to determine in a very simple way the Verblunsky coefficients $\Phi_{k}(0)$ of the measure $\sigma$ supported on the unit circle. Notice that the subsequence of the odd elements of the sequence $\left\{u_{k}\right\}_{k \geqslant 1}$ defined in (10) is the sequence $\left\{v_{k}\right\}_{k \geqslant 1}$ defined in (9).

Notice that, since the moments $\left\{c_{n}\right\}_{n \geqslant 0}$ are real, the Carathéodory function $F(z)$ associated with $\sigma$ has real coefficients. Therefore, we have

$$
\mathfrak{R e} F\left(e^{i \theta}\right)=\mathfrak{R e} F\left(e^{i(2 \pi-\theta)}\right),
$$

and then $d \sigma(\theta)+d \sigma(2 \pi-\theta)=0$. Thus, there is a relation between the Stieltjes and Carathéodory functions associated with $\mu$ and $\sigma$, respectively, as follows

$$
F(z)=\frac{1-z^{2}}{2 z} \int_{-1}^{1} \frac{d \mu(t)}{x-t}=\frac{1-z^{2}}{2 z} S(x)
$$

with $x=\frac{z+z^{-1}}{2}, z=x+\sqrt{x^{2}-1}($ see [12]).

\section{Spectral transformations on the real line and the unit circle: The connection through the Szegó transformation}

Our aim is to apply spectral rational transformations to the Stieltjes function $S(x)$ corresponding to the measure $\mu$ and to analyze the transformation obtained on the corresponding Carathéodory function $F(z)$ defined in the previous section. 


\subsection{Christoffel transformation}

Consider the Christoffel transformation of $S(x)$ given by

$$
\widetilde{S}(x)=\frac{(x-\beta) S(x)-1}{\mu_{1}-\beta},
$$

that represents the perturbation of the probability measure $\mu$ supported on $[-1,1]$

$$
d \tilde{\mu}=\frac{(x-\beta)}{\mu_{1}-\beta} d \mu,
$$

in such a way that $\tilde{\mu}$ is a probability measure.

Using (15) in (16)

$$
\begin{aligned}
\frac{2 z}{1-z^{2}} \widetilde{F}(z) & =\frac{\left(\frac{z+z^{-1}}{2}-\beta\right) \frac{2 z}{1-z^{2}} F(z)-1}{\mu_{1}-\beta} \\
\widetilde{F}(z) & =\frac{\frac{z+z^{-1}-2 \beta}{2} F(z)-\frac{1-z^{2}}{2 z}}{\mu_{1}-\beta} \\
& =\frac{\left(z^{2}-2 \beta z+1\right) F(z)+z^{2}-1}{2 z\left(\mu_{1}-\beta\right)}
\end{aligned}
$$

Then, the resulting transformation for Carathéodory functions can be represented by means of the linear rational transformation

$$
\widetilde{F}(z)=\frac{A(z) F(z)+B(z)}{D(z)},
$$

with $A(z)=\frac{z^{2}-2 \beta z+1}{2\left(\mu_{1}-\beta\right)}, B(z)=\frac{z^{2}-1}{2\left(\mu_{1}-\beta\right)}$, and $D(z)=z$.

In [8] it is shown that the canonical Christoffel transformation of a Carathéodory function is given by

$$
\widetilde{F}_{1}(z)=\frac{A_{1}(z) F_{1}(z)+B_{1}(z)}{D_{1}(z)},
$$

where $A_{1}(z)=-\bar{\alpha} z^{2}+\left(1+|\alpha|^{2}\right) z-\bar{\alpha}, B_{1}(z)=-\bar{\alpha} z^{2}+\left(\alpha c_{1}-\bar{\alpha} \bar{c}_{1}\right) z+\alpha$, and $D_{1}(z)=z$. Normalizing $\widetilde{F}_{1}(z)$ so that $\tilde{c}_{0}=1$, the polynomials on the above transformation become

$$
\begin{aligned}
& A_{1}(z)=\frac{-\bar{\alpha} z^{2}+\left(1+|\alpha|^{2}\right) z-\bar{\alpha}}{\left(1+|\alpha|^{2}\right)-2 \mathfrak{R e} \alpha c_{1}} \\
& B_{1}(z)=\frac{-\bar{\alpha} z^{2}+\left(\alpha c_{1}-\bar{\alpha} \bar{c}_{1}\right) z+\alpha}{\left(1+|\alpha|^{2}\right)-2 \mathfrak{R e} \alpha c_{1}} \\
& D_{1}(z)=z
\end{aligned}
$$


Thus, the comparison between the coefficients of $B(z)$ and $B_{1}(z)$ yields

$$
\alpha c_{1}-\bar{\alpha} c_{-1}=0 .
$$

So it follows that $\alpha \in \mathbb{R}$, and comparing the coefficients of $A(z)$ and the remaining coefficients of $B(z)$ with the coefficients of $A_{1}(z)$ and $B_{1}(z)$, respectively, we find:

$$
\frac{-\alpha}{\left(1+|\alpha|^{2}\right)-2 \alpha c_{1}}=\frac{1}{2\left(\mu_{1}-\beta\right)}
$$

and

$$
\frac{\left(1+\alpha^{2}\right)}{\left(1+|\alpha|^{2}\right)-2 \alpha c_{1}}=\frac{-2 \beta}{2\left(\mu_{1}-\beta\right)} .
$$

From (17),

$$
-2 \alpha\left(\mu_{1}-\beta\right)=\left(1+|\alpha|^{2}\right)-2 \alpha c_{1}
$$

and from (18) and (19)

$$
\frac{-\left(1+\alpha^{2}\right)}{2 \alpha\left(\mu_{1}-\beta\right)}=\frac{-2 \beta}{2\left(\mu_{1}-\beta\right)} .
$$

Then

$$
\alpha^{2}-2 \beta \alpha+1=0
$$

and, finally,

$$
\alpha_{ \pm}=\beta \pm \sqrt{\beta^{2}-1}, \text { with }|\beta| \geqslant 1 .
$$

This means that the zero of the complex polynomial of degree 1 in the canonical Christoffel transformation must be real.

\subsection{Uvarov transformation}

We now consider the Uvarov transformation of the Stieltjes function $S(x)$ associated with $\mu$

$$
\widetilde{S}(x)=\frac{S(x)+M_{r}(x-\beta)^{-1}}{1+M_{r}},
$$

corresponding to a transformation of the measure $\mu$ defined by

$$
d \tilde{\mu}=\frac{d \mu+M_{r} \delta_{\beta}}{1+M_{r}} .
$$

Again, $\tilde{\mu}$ is a probability measure. 
Using (15) and (20) we get

$$
\begin{aligned}
\frac{2 z}{1-z^{2}} \widetilde{F}(z) & =\frac{\frac{2 z}{1-z^{2}} F(z)+M_{r}\left(\frac{z+z^{-1}}{2}-\beta\right)^{-1}}{1+M_{r}} \\
\widetilde{F}(z) & =\frac{F(z)+M_{r}\left(\frac{2}{z+z^{-1}-2 \beta}\right)\left(\frac{1-z^{2}}{2 z}\right)}{1+M_{r}} \\
& =\frac{F(z)+M_{r}\left(\frac{1-z^{2}}{z^{2}-2 \beta z+1}\right)}{1+M_{r}} .
\end{aligned}
$$

In other words, $A(z)=\frac{z^{2}-2 \beta z+1}{1+M_{r}}, B(z)=\frac{\left(1-z^{2}\right) M_{r}}{1+M_{r}}$, and $D(z)=z^{2}-2 \beta z+1$.

On the other hand, from [8] we know that

$$
\widetilde{F}_{1}(z)=F(z)+\frac{B_{1}(z)}{D_{1}(z)},
$$

where $B_{1}(z)=\left(\alpha-\bar{\alpha} z^{2}\right)\left(M_{c}+\bar{M}_{c}\right)-\left(1-|\alpha|^{2}\right)\left(M_{c}-\bar{M}_{c}\right) z, D_{1}(z)=(z-\alpha)$ $(\bar{\alpha} z-1)$, and $F(z), \widetilde{F}_{1}(z)$ are the Carathéodory functions associated with the functions $\sigma$ and its Uvarov transformation $\tilde{\sigma}_{1}$, respectively, given by

$$
(p, q)_{\tilde{\sigma}_{1}}=(p, q)_{\sigma}+M_{c} p(\alpha) \overline{q\left(\bar{\alpha}^{-1}\right)}+\bar{M}_{c} p\left(\bar{\alpha}^{-1}\right) \overline{q(\alpha)}, \alpha \in \mathbb{C}-\{0\} .
$$

Taking into account

$$
\widetilde{F}_{1}(z)=\left(1+M_{c}+\bar{M}_{c}\right) F(z)
$$

we get

$$
M_{r}=2 \Re e M_{c} .
$$

On the other hand, the comparison between (23) and (24) yields

$$
\frac{\alpha-\bar{\alpha} z^{2}}{(z-\alpha)(\bar{\alpha} z-1)}=\frac{1-z^{2}}{z^{2}-2 \beta z+1}
$$

as well as

$$
\left(1-|\alpha|^{2}\right)\left(M_{c}-\bar{M}_{c}\right)=0 .
$$

As a consequence, two situations must be analyzed.

1. If $|\alpha|=1$, then from (25), $\frac{\alpha}{\bar{\alpha}}=1$ and $\beta=1$. This means that $\alpha_{ \pm}= \pm 1$. As a conclusion the Uvarov transformation of the measure $\sigma$ appears by the addition of one mass $M_{r}$ at the point $\alpha_{ \pm}= \pm 1$.

2. If $|\alpha| \neq 1$, then $M_{c} \in \mathfrak{R}$ and $M_{c}=\frac{M_{r}}{2}$. On the other hand, from (25), $\frac{\alpha}{\bar{\alpha}}=1$ as well as $\frac{1+\alpha^{2}}{\alpha}=2 \beta$, i.e. $\alpha=\bar{\alpha}$ and $\alpha^{2}-2 \beta \alpha+1=0$. This means that

$$
\alpha_{ \pm}=\beta \pm \sqrt{\beta^{2}-1}, \quad|\beta|>1 .
$$

As a conclusion, the Uvarov transformation of the measure $\sigma$ is the result of the addition of two real masses $\frac{M_{r}}{2}$ at the points $\alpha_{ \pm}=\beta \pm \sqrt{\beta^{2}-1}$, with $|\beta|>1$. 


\subsection{Geronimus transformation}

The Geronimus transformation of the Stieltjes function $S(x)$ associated with $\mu$ is given by

$$
\tilde{S}(x)=\frac{S(\beta)+M_{r}-S(x)}{(x-\beta)\left(M_{r}+S(\beta)\right)}
$$

and represents the following transformation of $\mu$

$$
d \tilde{\mu}=\frac{(x-\beta)^{-1} d \mu+M_{r} \delta_{\beta}}{M_{r}+S(\beta)} .
$$

Notice that $\tilde{\mu}$ is a probability measure. The corresponding Carathéodory function $\widetilde{F}(z)$ can be obtained from

$$
\begin{aligned}
\frac{2 z}{1-z^{2}} \widetilde{F}(z) & =\frac{S(\beta)+M_{r}-\frac{2 z}{1-z^{2}} F(z)}{\left(\frac{z+z^{-1}}{2}-\beta\right)\left(M_{r}+S(\beta)\right)} \\
\widetilde{F}(z) & =\frac{-2 z F(z)+\left(1-z^{2}\right)\left[S(\beta)+M_{r}\right]}{\left(z^{2}-2 \beta z+1\right)\left(S(\beta)+M_{r}\right)},
\end{aligned}
$$

or, equivalently,

$$
\widetilde{F}(z)=\frac{z F(z)+\frac{1}{2}\left(z^{2}-1\right)\left[S(\beta)+M_{r}\right]}{-\frac{1}{2}\left(z^{2}-2 \beta z+1\right)\left[S(\beta)+M_{r}\right]} .
$$

Thus, we have $A(z)=z, B(z)=\frac{1}{2}\left(z^{2}-1\right)\left[S(\beta)+M_{R}\right]$, and $D(z)=-\frac{1}{2}\left(z^{2}-\right.$ $2 \beta z+1)\left[S(\beta)+M_{r}\right]$. On the other hand, in [8] it is shown that

$$
\widetilde{F}_{1}(z)=\frac{A_{1}(z) F(z)+B_{1}(z)}{D_{1}(z)}
$$

where

$$
\begin{aligned}
& A_{1}(z)=z \\
& B_{1}(z)=\bar{\alpha} z^{2}-2 i \Im \mathfrak{I m}\left(q_{0}\right) z-\alpha, \\
& D_{1}(z)=-\bar{\alpha} z^{2}+\left(1+|\alpha|^{2}\right) z-\alpha,
\end{aligned}
$$

and $q_{0}$ is a free parameter defined by

$$
q_{0}=\tilde{c}_{0}-\bar{\alpha} \tilde{c}_{1} .
$$

Then, comparing the coefficients from $B(z)$ and $B_{1}(z)$, we get $q_{0} \in \mathbb{R}$. On the other hand, (27) can also be expressed as

$$
\widetilde{F}_{1}(z)=\frac{A_{1}(z)}{D_{1}(z)} F(z)+M_{c} \frac{\alpha+z}{\alpha-z}+\bar{M}_{c} \frac{1+\bar{\alpha} z}{1-\bar{\alpha} z}
$$

with $M_{c}=\frac{\overline{q_{0}}-\frac{1}{2}}{1-|\alpha|^{2}}$. Therefore, $M_{c} \in \mathbb{R}$ and (28) becomes

$$
\widetilde{F}_{1}(z)=\frac{z F(z)-2 M_{c}\left(-\bar{\alpha} z^{2}+\alpha\right)}{\left(-\bar{\alpha} z^{2}+\left(1+|\alpha|^{2}\right) z-\alpha\right) A_{g}}
$$


where $A_{g}$ is the normalization factor. Comparing the coefficients of the polynomials on (26) and (29) we get

$$
\alpha \in \mathbb{R}, \quad M_{c}=\frac{S(\beta)+M_{r}}{4 \alpha},
$$

and

$$
\alpha^{2}-2 \beta \alpha+1=0
$$

Then,

$$
\alpha_{ \pm}=\beta \pm \sqrt{\beta^{2}-1}, \quad \text { with } \quad|\beta|>1 .
$$

This is, in the canonical Geronimus transformation, two real masses are added at points $\alpha_{ \pm}=\beta \pm \sqrt{\beta^{2}-1}$.

\section{Examples}

\subsection{Jacobi case}

We consider the family of orthogonal polynomials corresponding to the measure

$$
d \mu=(1-x)^{\alpha}(1+x)^{\beta} d x, \quad \alpha, \beta>-1,
$$

supported on the interval $(-1,1)$.

It is well known (see $[2,14]$ ) that the coefficients of the three-term recurrence relation are given by

$$
\begin{aligned}
& b_{n}=\frac{\beta^{2}-\alpha^{2}}{(2 n+\alpha+\beta)(2 n+2+\alpha+\beta)}, \quad n \geqslant 0, \\
& a_{n}^{2}=\frac{4 n(n+\alpha)(n+\beta)(n+\alpha+\beta)}{(2 n+\alpha+\beta-1)(2 n+\alpha+\beta)^{2}(2 n+\alpha+\beta+1)}, \quad n \geqslant 1 .
\end{aligned}
$$

We analyze some representative Jacobi cases.

\subsubsection{Tchebichef polynomials of first kind, $\left(\alpha=\beta=-\frac{1}{2}\right)$}

In this case

$$
b_{n}=0, n \geqslant 0, \quad a_{1}^{2}=\frac{1}{2}, \quad \text { and } \quad a_{n}^{2}=\frac{1}{4}, n \geqslant 2 .
$$

Therefore,

$$
\tilde{J}+I=\left(\begin{array}{ccccc}
1 & 1 & 0 & 0 & \cdots \\
\frac{1}{2} & 1 & 1 & 0 & \cdots \\
0 & \frac{1}{4} & 1 & 1 & \ddots \\
0 & 0 & \frac{1}{4} & 1 & \ddots \\
\vdots & \vdots & \ddots & \ddots & \ddots
\end{array}\right)
$$


From the $L U$ factorization, we obtain

$$
u_{1}=1 \quad \text { and } \quad u_{n}=\frac{1}{2}, \quad n \geqslant 2,
$$

and from (13)

$$
\Phi_{n}(0)=0, \quad n \geqslant 1,
$$

as expected, since the measure on the unit circle associated with (30) for $\alpha=\beta=-\frac{1}{2}$ is the normalized Lebesgue measure.

5.1.2 Tchebichev polynomials of second kind, $\left(\alpha=\beta=\frac{1}{2}\right)$

In this case,

$$
b_{n}=0, n \geqslant 0, \quad \text { and } \quad a_{n}^{2}=\frac{1}{4}, n \geqslant 1
$$

Therefore, $\tilde{J}+I$ becomes

$$
\tilde{J}+I=\left(\begin{array}{ccccc}
1 & 1 & 0 & 0 & \cdots \\
\frac{1}{4} & 1 & 1 & 0 & \cdots \\
0 & \frac{1}{4} & 1 & 1 & \ddots \\
0 & 0 & \frac{1}{4} & 1 & \ddots \\
\vdots & \vdots & \ddots & \ddots & \ddots
\end{array}\right)
$$

Calculating the $L U$ factorization of this matrix, we obtain, for the elements of the sequence $\left\{u_{n}\right\}_{n} \geqslant 1$,

$$
\begin{aligned}
u_{2 n} & =\frac{2 n}{4(n+1)}, \quad n \geqslant 1, \\
u_{2 n+1} & =\frac{n+2}{2(n+1)}, \quad n \geqslant 0
\end{aligned}
$$

and from (13) we get

$$
\begin{array}{cc}
\Phi_{2 n}(0)=\frac{1}{n+1}, & n \geqslant 1, \\
\Phi_{2 n+1}(0)=0, & n \geqslant 0 .
\end{array}
$$

This is a perturbation of the Lebesgue measure given by

$$
d \tilde{\mu}=\left|z^{2}-1\right|^{2} \frac{d \theta}{2 \pi}, \quad z=e^{i \theta} .
$$

Notice that the measure $\tilde{\mu}$ is a sieved measure of $d \mu_{1}=|z-1|^{2} \frac{d \theta}{2 \pi}, z=e^{i \theta}$. Sieved orthogonal polynomials relative to measures supported on the unit circle have been analyzed in [7] and [11]. 
5.1.3 Tchebichev polynomials of third kind $\left(\alpha=\frac{1}{2}, \beta=-\frac{1}{2}\right)$

We get

$$
b_{0}=-\frac{1}{2}, \quad b_{n}=0, n \geqslant 1, \quad \text { and } \quad a_{n}^{2}=\frac{1}{4}, n \geqslant 1,
$$

so that

$$
\tilde{J}+I=\left(\begin{array}{ccccc}
\frac{1}{2} & 1 & 0 & 0 & \cdots \\
\frac{1}{4} & 1 & 1 & 0 & \cdots \\
0 & \frac{1}{4} & 1 & 1 & \ddots \\
0 & 0 & \frac{1}{4} & 1 & \ddots \\
\vdots & \vdots & \ddots & \ddots & \ddots
\end{array}\right)
$$

Calculating the $L U$ factorization of this matrix, we obtain

$$
u_{k}=\frac{1}{2}, \quad k \geqslant 1,
$$

and from (13) we deduce

$$
\Phi_{n}(0)=\frac{1}{n+1}, \quad n \geqslant 1 .
$$

This corresponds to a perturbation of the Lebesgue measure given by (see [10])

$$
d \tilde{\mu}=|z-1|^{2} \frac{d \theta}{2 \pi}, \quad z=e^{i \theta} .
$$

Indeed, it is a canonical transform of the Lebesgue measure as pointed out in Section 4.1, with $\alpha=1$.

5.1.4 Tchebichev polynomials of fourth kind $\left(\alpha=-\frac{1}{2}, \beta=\frac{1}{2}\right)$

We get

$$
b_{0}=\frac{1}{2}, \quad b_{n}=0, n \geqslant 1, \quad \text { and } \quad a_{n}^{2}=\frac{1}{4}, n \geqslant 1,
$$

so that

$$
\tilde{J}+I=\left(\begin{array}{ccccc}
\frac{3}{2} & 1 & 0 & 0 & \cdots \\
\frac{1}{4} & 1 & 1 & 0 & \cdots \\
0 & \frac{1}{4} & 1 & 1 & \ddots \\
0 & 0 & \frac{1}{4} & 1 & \ddots \\
\vdots & \vdots & \ddots & \ddots & \ddots
\end{array}\right)
$$


Calculating the $L U$ factorization, we obtain for the elements of the sequence $\left\{u_{n}\right\}_{n \geqslant 1}$

$$
\begin{aligned}
u_{2 n} & =\frac{2 n-1}{4 n+2}, \quad n \geqslant 1, \\
u_{2 n+1} & =\frac{2 n+3}{4 n+2}, \quad n \geqslant 0
\end{aligned}
$$

and from (13) we deduce

$$
\Phi_{n}(0)=\frac{(-1)^{n}}{n+1}, \quad n \geqslant 1
$$

This corresponds to a perturbation of the Lebesgue measure given by (see $[5,10])$

$$
d \tilde{\mu}=|z+1|^{2} \frac{d \theta}{2 \pi}, \quad z=e^{i \theta} .
$$

Indeed, it is a canonical Christoffel transformation of the Lebesgue measure as pointed out in Section 4.1, with $\alpha=-1$.

5.1.5 Gegenbauer polynomials, with $\alpha=\beta=1$

In this case

$$
b_{n}=0, n \geqslant 0, \quad a_{n}^{2}=\frac{n(n+2)}{(2 n+1)(2 n+3)}, n \geqslant 1 .
$$

Therefore, from the $L U$ factorization of $\tilde{J}+I$ we obtain

$$
\begin{aligned}
u_{2 n} & =\frac{n}{2 n+3}, \quad n \geqslant 1, \\
u_{2 n+1} & =\frac{n+3}{2 n+3}, \quad n \geqslant 0,
\end{aligned}
$$

and from (13) we get

$$
\begin{array}{ccc}
\Phi_{2 n}(0) & =\frac{3}{2 n+3}, & n \geqslant 1, \\
\Phi_{2 n+1}(0) & =0, & n \geqslant 1 .
\end{array}
$$

This corresponds to a perturbation of the Lebesgue measure defined by

$$
d \tilde{\mu}=\left|z^{2}-1\right|^{3} \frac{d \theta}{2 \pi}, \quad z=e^{i \theta} .
$$

5.2 Uvarov transformation

We consider the Legendre case $d \mu=d x$, i.e., a Jacobi case with $\alpha=\beta=0$, and its Uvarov transformation defined by

$$
d \tilde{\mu}=d x+\delta(x+1)+\delta(x-1)
$$


We denote $J$ the monic Jacobi matrix associated with $\mu$. From Corollary 4.5 on [1], if we apply the following transformations

$$
\begin{aligned}
J-I & =L_{1} U_{1} \\
J_{1} & :=U_{1} L_{1} \\
J_{1} & =U_{2} L_{2} \\
J_{2} & :=L_{2} U_{2}+I \\
J_{2}+I & =L_{3} U_{3} \\
J_{3} & :=U_{3} L_{3} \\
J_{3} & =U_{4} L_{4} \\
J_{4} & :=L_{4} U_{4}-I
\end{aligned}
$$

then $J_{4}$ is the monic Jacobi matrix associated with (31). We now calculate the $L U$ factorization of $J_{4}+I$ to obtain the sequence $\left\{u_{k}\right\}_{n \geqslant 1}$, such that

$$
\begin{aligned}
u_{2 n} & =\frac{(n+1)^{2}\left[n^{2}(2 n+4)+4 n\right]+2 n}{2(2 n+1)\left[n^{2}(n+1)^{2}+2 n(n+1)+1\right]}, \quad n \geqslant 1, \\
u_{2 n+1} & =\frac{n^{2}(n+1)^{2}(2 n-2)+(n+1)\left(4 n^{2}+2\right)}{2(2 n+1)\left[n^{2}(n+1)^{2}+2 n(n+1)+1\right]}, \quad n \geqslant 0 .
\end{aligned}
$$

From (13) we get

$$
\begin{gathered}
\Phi_{2 n}(0)=-\frac{3 n^{2}(n+1)^{2}+2 n(n+1)-1}{(2 n+1)\left[n^{2}(n+1)^{2}+2 n(n+1)+1\right]}, \quad n \geqslant 1, \\
\Phi_{2 n+1}(0)=0, \quad n \geqslant 1 .
\end{gathered}
$$

Acknowledgements We thank the valuable comments and suggestions by the referee in order to improve the presentation of the manuscript. The work of the first author (L. Garza) was supported by a grant of Universidad Autónoma de Tamaulipas. The research of the third author (F. Marcellán) has been supported by Dirección General de Investigación, Ministerio de Educación y Ciencia of Spain, grant MTM 2006-13000-C03-02.

\section{References}

1. Bueno, M.I., Marcellán, F.: Darboux transformations and perturbation of linear functionals. Linear Alg. Appl. 384, 215-242 (2004)

2. Chihara, T.S.: An Introduction to Orthogonal Polynomials. Gordon and Breach, New York (1978)

3. Daruis, L., Hernández, J., Marcellán, F.: Spectral transformations for Hermitian Toeplitz matrices. J. Comput. Appl. Math. 202, 155-176 (2007)

4. Garza, L., Hernández, J., Marcellán, F.: Orthogonal polynomials and measures on the unit circle. The Geronimus transformations. J. Comput. Appl. Math. (in press)

5. Geronimus, Ya.L.: Polynomials orthogonal on a circle and their applications. Amer. Math. Soc. Transl. 1, 1-78, Providence, RI (1962)

6. Godoy, E., Marcellán, F.: Orthogonal polynomials and rational modification of measures. Canada J. Math. 45, 930-943 (1993) 
7. Ismail, M.E.H., Li, X.: On sieved orthogonal polynomials IX: orthogonality on the unit circle, Pacific J. Math. 153, 289-297 (1992)

8. Marcellán, F.: Polinomios ortogonales no estándar. Aplicaciones en análisis numérico y teoría de aproximación. Rev. Acad. Colomb. Ciencias Exactas, Físicas y Naturales, 29(117), 1-17 (2006) (in Spanish)

9. Marcellán, F., Hernández, J.: Geronimus spectral transforms and measures on the complex plane. J. Comput. Appl. Math. (in press)

10. Marcellán, F., Hernández, J.: Christoffel transforms and Hermitian linear functionals. Mediterr. J. Math. 2, 451-458 (2005)

11. Marcellán, F., Sansigre, G.: Symmetrization, quadratic decomposition and cubic transformations of orthogonal polynomials on the unit circle. In: Brezinski, C. et al. (eds.) Proceedings Erice International Symposium on Orthogonal Polynomials and Their Applications. IMACS Annals on Comput. and Appl. Math. pp. 341-345 (1991)

12. Peherstorfer, F.: A special class of polynomials orthogonal on the unit circle including the associated polynomials. Constr. Approx. 12, 161-185 (1996)

13. Simon, B.: Orthogonal polynomials on the unit circle, two volumes. Amer. Math. Soc. Colloq. Publ. Series, vol. 54, Amer. Math. Soc. Providence, RI (2005)

14. Szegó, G.: Orthogonal Polynomials, $4^{\text {th }}$ ed., Amer. Math. Soc. Colloq. Publ. Series, vol. 23, Amer. Math. Soc., Providence, RI (1975)

15. Zhedanov, A.: Rational spectral transformations and orthogonal polynomials. J. Comput. Appl. Math. 85, 67-83 (1997) 